\title{
Classroom Models for Illustrating Dynamics Principles Part II. - Rigid Body Kinematics and Kinetics
}

\author{
Michael A. Magill, Ph.D., P.E. \\ Purdue University
}

\begin{abstract}
This paper is part II in a two part series that describes a collection of ten classroom models used to illustrate basic Dynamics principles. The models discussed in part I of the series cover the topics of Particle Kinematics and Kinetics while part II covers Rigid Body Kinematics and Kinetics. These models are excellent tools for communicating basic Engineering Mechanics concepts while also stimulating interest and enthusiasm. These devices were developed for undergraduate engineering technology students but they are equally valuable for engineering students. Most of these models are inexpensive or can be constructed easily.
\end{abstract}

\section{INTRODUCTION}

Dynamics is one of the more difficult courses that engineering and engineering technology students encounter during their undergraduate study. As a result, mechanics instructors are trying continually to find or develop techniques that enhance student learning. One of the greatest challenges is creating student interest and enthusiasm. It is well known that students learn more and work harder when they are interested in a topic. A good technique for breaking the monotony of classroom lectures and creating student interest is to introduce exciting classroom models. These models teach basic mechanics principles but more importantly they get students involved, stimulate interest and give a change of pace. The time required to properly present a model is roughly the same as presenting an example problem.

\section{THE CLASSROOM MODELS}

The models discussed in this paper (Part II) cover the topics of Rigid Body Kinematics and Kinetics. All the information necessary for developing these models and presenting them in the classroom is provided within the paper. The details for each model are provided on separate pages to facilitate duplicating and using them as classroom handouts. The description of each model includes an interesting problem statement, descriptive diagrams, and the analytical solution.

The five classroom models* presented in this paper are:

-Equation of Motion - Rolling Yo-Yo

-Equation of Motion - Rolling Ring Versus Rolling Disk

-Mass Moment of Inertia - Rolling Solid Cylinder Versus Rolling Hollow Cylinder

-Work/Energy - Weight Suspended From a Wheel

-Conservation of Momentum - Rotating Bicycle Wheel 


\section{PROCEDURE/APPROACH}

The approach used for presenting these models will control their ability to stimulate interest and communicate mechanics principles. Just showing the model and throwing the analytical solution on the overhead will not produce the desired results. The following is an outlined approach that the author has found to be successful.

- Start by introducing the model and posing one or two interesting questions.

- Ask for a show of hands on each of the possible solutions. Establish a competitive spirit in the classroom. Have a couple students offer a solution using their "gut feeling."

- The next step is to lead the students through the analytical solution. This phase should be performed quickly without covering a lot of detail. Too much detail at this point will distract the students and they may lose interest. This phase moves more efficiently and is more effective if copies of the analytical solution are distributed and also shown on the overhead. This phase of reviewing the analytical solution can include the following:

- Determine if the problem requires a particle or rigid body solution. (i.e., Is the body rotating? Is the body's size of consequence? Make a clear distinction between rotation and curvilinear motion.)

- Determine if the problem requires a kinematic or kinetic solution or both. (i.e., Does this problem involve a force analysis or just motion geometry?)

- If the solution requires a kinetic solution determine which procedure is best.

- Establish the proper diagrams.

- Set up the basic governing equation(s).

- Obtain one or two volunteers to assist with demonstrating the model. Make the demonstration fun and interesting.

- Return to the analytical solution to clear up details and ask additional questions. This is also a good time to discuss the validity of any assumptions.

There are many creative techniques for using models. Try having students present the models. Better yet, have each student develop a model and then present it and the analytical solution to the class. The best student models can be saved for future semesters.

\section{CONCLUSION}

The key to making these models successful is making them fun and interesting. Models are excellent tools for communicating basic Engineering Mechanics concepts while also stimulating interest and enthusiasm.

MICHAEL MAGILL is an Associate Professor in Mechanical Engineering Technology at Purdue University. Prior to joining Purdue University he was on the faculty at Oklahoma State University for eleven years. Professor Magill's areas of interest are solid mechanics, applied structural analysis and computer-aided analysis. He has a B.S. and M.S. in Mechanical Engineering and a Ph.D. in Civil Engineering, all from Oklahoma State University.

*The ideas for these models came from various sources so the author does not claim ownership or copyright. 


\section{RIGID BODY: EQUATION OF MOTION - ROLLING YO-YO}

Supplies: Yo-yo.

Given:

$r_{1}=$ yo-yo center peg radius

$r_{2}=$ yo-yo radius

$m=$ yo-yo mass

$T=$ constant string tension

Assume no slipping!

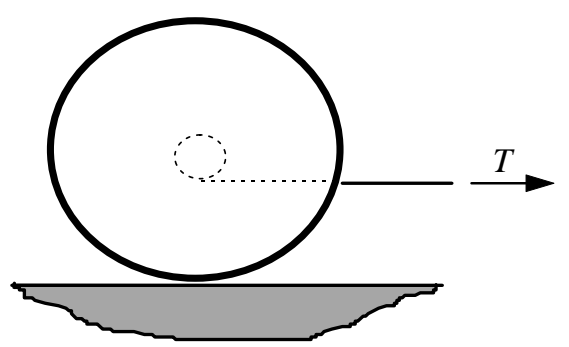

(a)

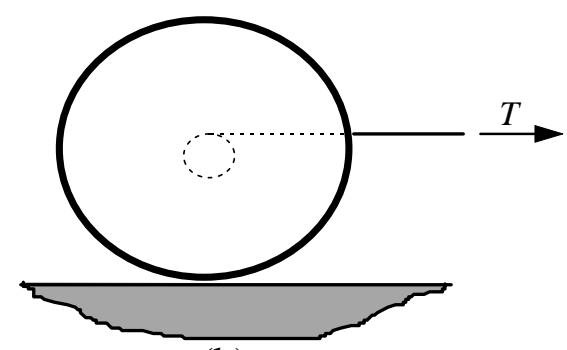

(b)

Find: Which way will yo-yo roll?

Solution:

a) Assume yo-yo moves to the right so $m a_{\mathrm{G}}$ must be to the right and $F_{\mathrm{f}}$ to the left to force rolling.

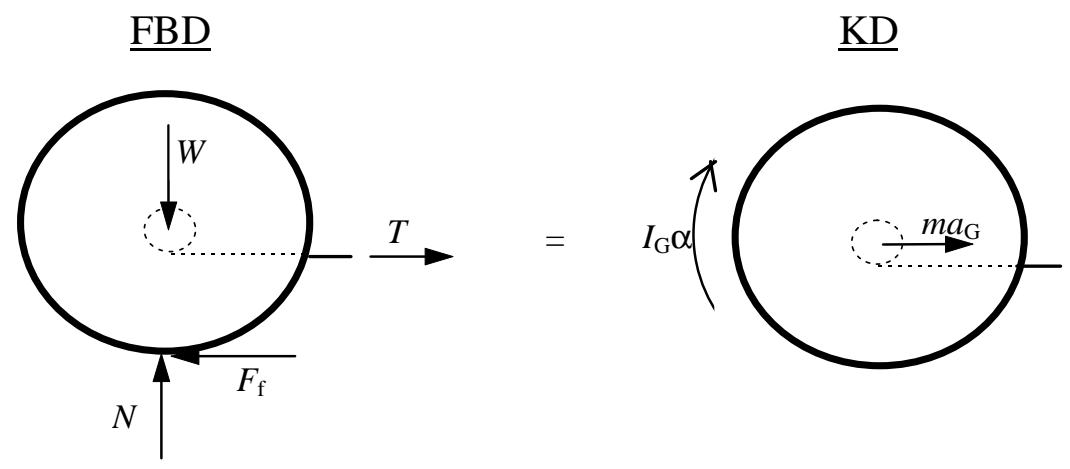

$\Sigma F_{\mathrm{x}}=T-F_{\mathrm{f}}=m a_{\mathrm{G}} \quad \leftarrow T$ must be greater than $F_{\mathrm{f}}$ or $m a_{\mathrm{G}}$ will be to the left.

$T-F_{\mathrm{f}}=m \alpha r_{2} \quad \leftarrow a_{\mathrm{G}}=\alpha r_{2}$ because there is no slipping.

$\alpha=\frac{T-F_{\mathrm{f}}}{m r_{2}} \quad \leftarrow$

$\therefore$ Positive, so correct direction assumed!! 
Try the problem again assuming yo-yo moves to the left; so $m a_{\mathrm{G}}$ must be to the left and $F_{\mathrm{f}}$ to the right to force rolling.

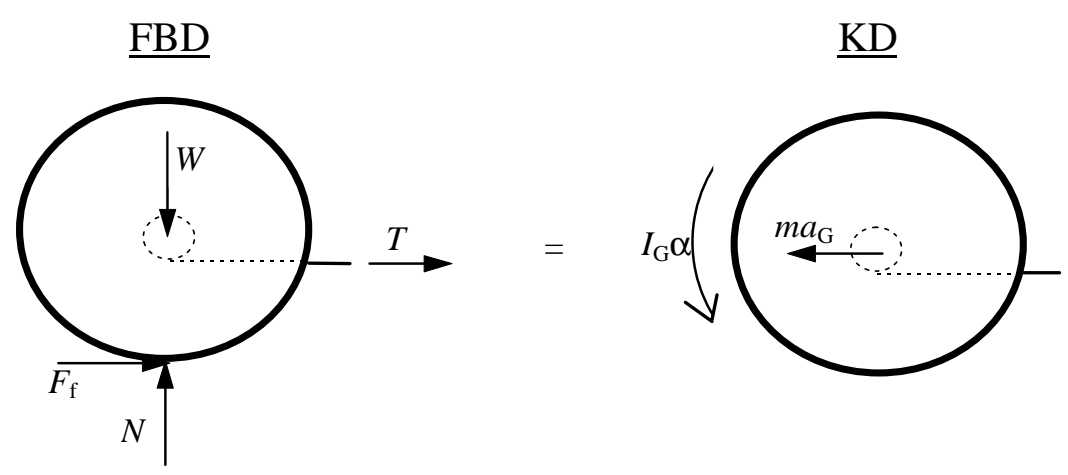

$$
\begin{aligned}
& \Sigma F_{\mathrm{x}}=T+F_{\mathrm{f}}=-m a_{\mathrm{G}} \\
& T+F_{\mathrm{f}}=-m \alpha r_{2} \\
& \alpha=-\frac{T+F_{\mathrm{f}}}{m r_{2}}
\end{aligned}
$$

$\therefore$ Negative, so wrong direction assumed!!

b) Assume yo-yo moves to the right so $m a_{\mathrm{G}}$ must be to the right and $F_{\mathrm{f}}$ to the left to force rolling.

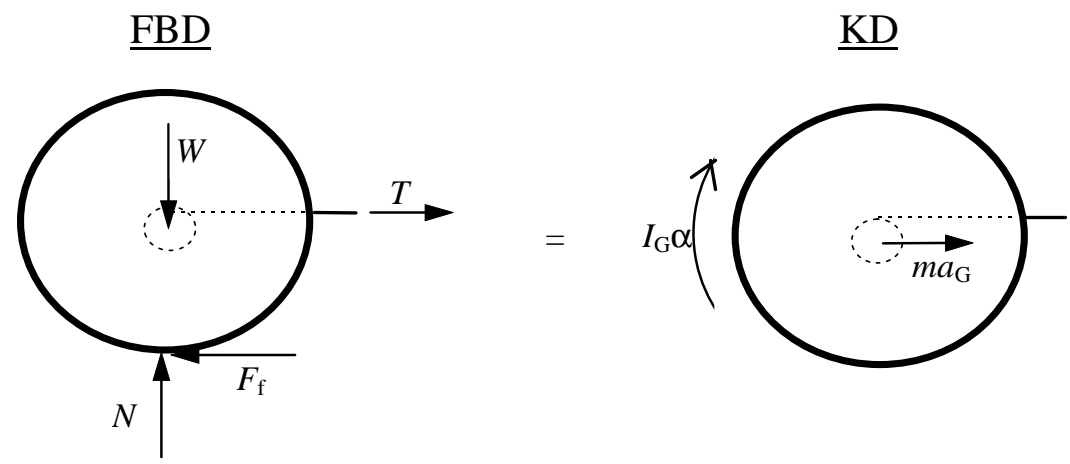

$$
\begin{aligned}
& \Sigma F_{\mathrm{x}}=T-F_{\mathrm{f}}=m a_{\mathrm{G}} \\
& T-F_{\mathrm{f}}=m \alpha r_{2} \\
& \alpha=\frac{T-F_{\mathrm{f}}}{m r_{2}} \leftarrow
\end{aligned}
$$

$\therefore$ Positive, so correct direction assumed!! 


\section{RIGID BODY: EQUATION OF MOTION - ROLLING RING VERSUS ROLLING DISK}

Supplies: Disk and ring with the same weight and outside diameter. A good example is a wooden disk and a steel ring. Machine the outside diameters to about 6 inches and the thicknesses to about 1.5 inches. Machine the inside diameter of the ring until both the ring and disk weigh the same. The wall thickness of the ring will be about 0.125 inches.

Given:

$\underline{\text { Disk }}$

$m_{\mathrm{D}}=m_{\mathrm{R}}=m=$ mass

$\theta_{\mathrm{D}}=\theta_{\mathrm{R}}=\theta$

$r_{\mathrm{D}}=r_{\mathrm{R}}=r$

Assume no slipping
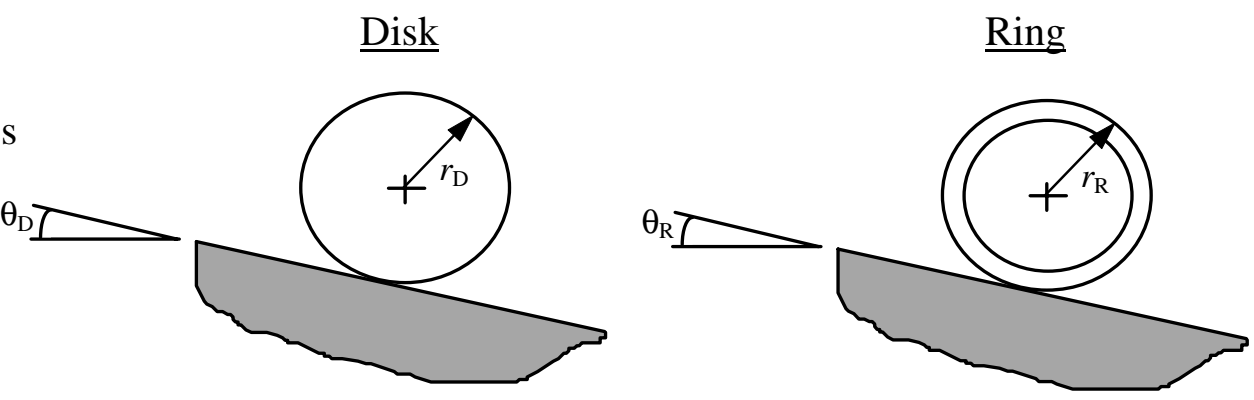

Find: Which will roll faster, disk or ring?

Solution:

$\underline{\text { FBD }}$

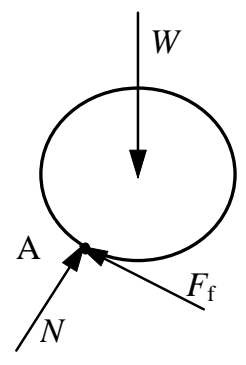

$\underline{\mathrm{KD}}$

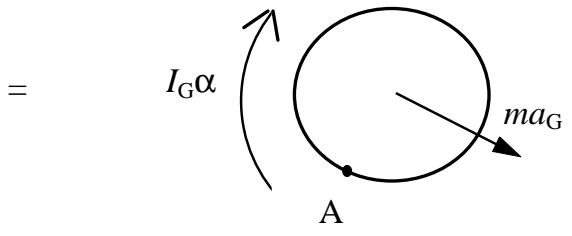

$\leftarrow a_{\mathrm{G}}=\alpha r$

$\leftarrow I_{\mathrm{A}}=I_{\mathrm{G}}+m r^{2}$

$W r \sin \theta=\alpha\left(I_{\mathrm{G}}+m r^{2}\right)$

$W r \sin \theta=\alpha I_{\mathrm{A}}$

$\alpha=\frac{W r \sin \theta}{I_{\mathrm{A}}}$

$\therefore$ The one with the smaller $I_{\mathrm{A}}$ will roll faster!

$I_{\mathrm{A}_{\mathrm{DISK}}}=I_{\mathrm{G}_{\mathrm{DISK}}}+m d^{2}$

$I_{\mathrm{A}_{\mathrm{DISK}}}=\frac{1}{2} m r^{2} m(r)^{2}$

$I_{\mathrm{A}_{\mathrm{DISK}}}=\frac{3}{2} m r^{2}$

$$
\begin{aligned}
& I_{\mathrm{A}_{\mathrm{RING}}}=I_{\mathrm{G}_{\mathrm{RING}}}+m d^{2} \\
& I_{\mathrm{A}_{\mathrm{RING}}}=m r^{2}+m(r)^{2} \\
& I_{\mathrm{A}_{\mathrm{RING}}}=2 m r^{2}
\end{aligned}
$$

$$
I_{\mathrm{A}_{\mathrm{DISK}}}<I_{\mathrm{A}_{\mathrm{RING}}} \quad \therefore \text { Disk will roll faster! }
$$




\section{RIGID BODY: MASS MOMENT OF INERTIA - ROLLING SOLID CYLINDER VERSUS ROLLING HOLLOW CYLINDER}

Supplies: Solid cylinder and hollow cylinder make from the same material and having the same outside diameter.

Given:

$R_{\mathrm{S}}=R_{\mathrm{H}}=R$

$\theta_{\mathrm{S}}=\theta_{\mathrm{H}}=\theta$

$C=r / R$

$m_{\mathrm{H}}=$ mass of hollow cylinder

$m_{\mathrm{S}}=$ mass of solid cylinder

$m_{\text {Hole }}=$ mass of hollow cylinder hole

$\rho_{\mathrm{S}}=\rho_{\mathrm{H}}=\rho=$ mass density

$V_{\mathrm{H}}=$ volume of hollow cylinder

$V_{\mathrm{S}}=$ volume of solid cylinder

$V_{\text {Hole }}=$ volume of hollow cylinder hole

Assume no slipping

Find: Which cylinder will roll faster, solid or hollow?

Solution: $\underline{\text { FBD }}$

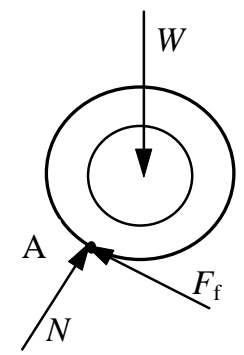

$\Sigma M_{\mathrm{A}}=-W R \sin \theta=-I_{\mathrm{G}} \alpha-m_{\mathrm{H}} a_{\mathrm{G}} R$

$$
W R \sin \theta=\left(I_{\mathrm{G}}+m_{\mathrm{H}} R^{2}\right) \alpha
$$$$
\alpha=\frac{W R \sin \theta}{I_{\mathrm{G}}+m_{\mathrm{H}} R^{2}}
$$$$
\alpha=\frac{\left(m_{\mathrm{H}} g\right) R \sin \theta}{I_{\mathrm{G}}+m_{\mathrm{H}} R^{2}}
$$

$\underline{\text { Solid }}$

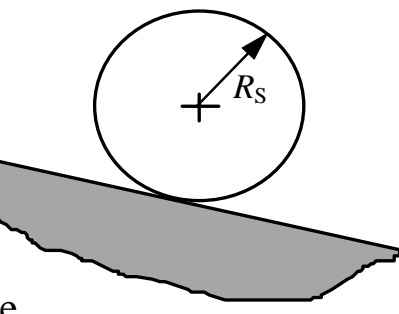

Hollow

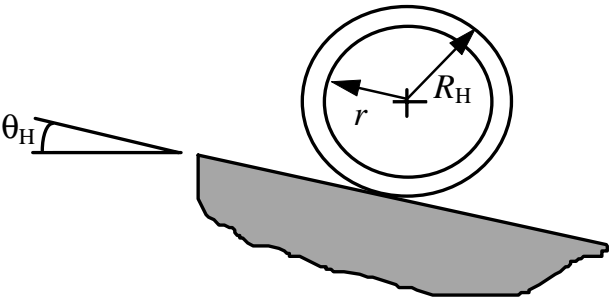

$\underline{\mathrm{KD}}$

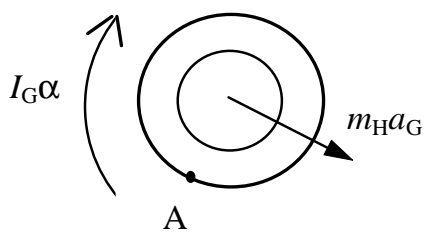

$\leftarrow a_{\mathrm{G}}=\alpha R$

$\leftarrow W=m_{\mathrm{H}} g$

$\leftarrow I_{\mathrm{G}}=\frac{1}{2} m_{\mathrm{S}} R^{2}-\frac{1}{2} m_{\mathrm{Hole}} r^{2}$ 


$$
\begin{aligned}
& \alpha=\frac{\left(m_{\mathrm{H}} g\right) R \sin \theta}{\left(\frac{1}{2} m_{\mathrm{S}} R^{2}-\frac{1}{2} m_{\mathrm{Hole}} r^{2}\right)+m_{\mathrm{H}} R^{2}} \\
& \alpha=\frac{\left(\rho V_{\mathrm{H}} g\right) R \sin \theta}{\frac{1}{2} \rho V_{\mathrm{S}} R^{2}-\frac{1}{2} \rho V_{\text {Hole }}(C R)^{2}+\rho V_{\mathrm{H}} R^{2}} \\
& \alpha=\frac{\left[\pi\left(R^{2}-(C R)^{2}\right) L\right] g R \sin \theta}{\frac{1}{2}\left(\pi R^{2} L\right) R^{2}-\frac{1}{2}\left(\pi(C R)^{2} L\right)(C R)^{2}+\left[\pi\left(R^{2}-(C R)^{2}\right) L\right] R^{2}} \leftarrow \pi \text { and } L \text { factor out } \\
& \alpha=\frac{\left[R^{2}-C^{2} R^{2}\right] g R \sin \theta}{\frac{1}{2}\left(R^{2}\right) R^{2}-\frac{1}{2}\left(C^{2} R^{2}\right)\left(C^{2} R^{2}\right)+\left[R^{2}-\left(C^{2} R^{2}\right)\right] R^{2}} \\
& \alpha=\frac{R^{3}\left(1-C^{2}\right) g \sin \theta}{\frac{1}{2} R^{4}-\frac{1}{2} R^{4} C^{4}+R^{4}\left(1-C^{2}\right)} \\
& \alpha=\frac{R^{3}\left(1-C^{2}\right) g \sin \theta}{R^{4}\left(\frac{1}{2}-\frac{1}{2} C^{4}+1-C^{2}\right)} \\
& \alpha=\frac{\left(1-C^{2}\right) g \sin \theta}{R\left(\frac{3}{2}-C^{2}-\frac{1}{2} C^{4}\right)} \\
& \alpha=\frac{\left(1-C^{2}\right) g \sin \theta}{R\left(\frac{3}{2}+\frac{1}{2} C^{2}\right)\left(1-C^{2}\right)} \\
& \alpha=\frac{g \sin \theta}{R\left(\frac{3}{2}+\frac{1}{2} C^{2}\right)} \\
& \alpha=\frac{g \sin \theta}{2 R\left(3+C^{2}\right)} \\
& \alpha=\frac{g \sin \theta}{2 R\left(3+\left(\frac{r}{R}\right)^{2}\right)}
\end{aligned}
$$




\section{RIGID BODY: WORK/ENERGY - WEIGHT SUSPENDED FROM A WHEEL}

Supplies: Bicycle wheel, string, and tennis ball.

Given:

$r=$ radius of bicycle wheel

$I_{\mathrm{G}}=$ moment of inertia of wheel

$m_{\mathrm{B}}=$ mass of tennis ball

$W_{\mathrm{W}}=$ weight of bicycle wheel

$W_{\mathrm{B}}=$ weight of tennis ball

$\omega_{2}=$ final angular velocity of wheel

$v_{2}=$ final velocity of tennis ball

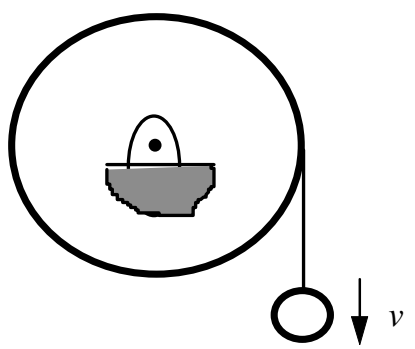

(a)

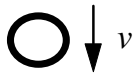

(b)

Find: How long does it take for the tennis ball to fall a distance $y$ starting from rest?

a) Attached to bicycle wheel.

b) Free fall.

Solution:

a)

$\underline{\text { FBD }}$

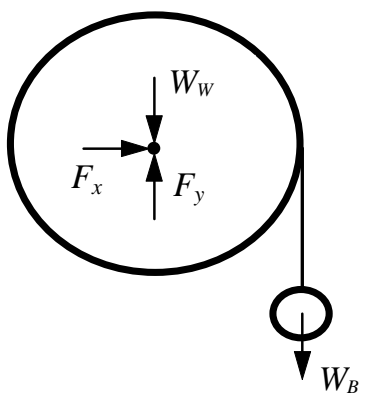

$\underline{\text { Kinematic Diagram }}$

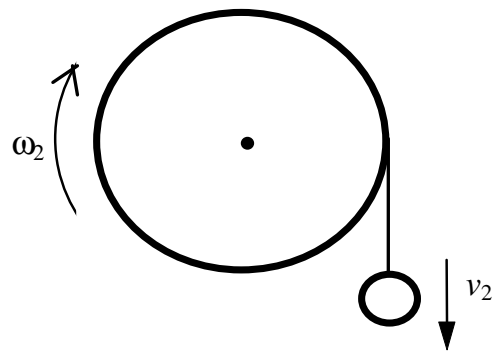

$\leftarrow U_{\mathrm{WB}}=W_{\mathrm{B}} y$ and $\omega_{2}=v_{2} / \mathrm{r}$

$T_{1}+\Sigma U_{1-2}=T_{2}$

$1 / 2 m_{\mathrm{B}} v_{1}^{2}+1 / 2 I_{\mathrm{G}} \omega_{1}^{2}+U_{\mathrm{WB}}=1 / 2 m_{\mathrm{B}} v_{2}^{2}+1 / 2 I_{\mathrm{G}} \omega_{2}^{2}$

$0+0+W_{\mathrm{B}} y=1 / 2 m_{\mathrm{B}} v_{2}^{2}+1 / 2 I_{\mathrm{G}}\left(v_{2} / \mathrm{r}\right)^{2}$

$2 W_{\mathrm{B}} y=v_{2}^{2}\left(m_{\mathrm{B}}+I_{\mathrm{G}} / \mathrm{r}^{2}\right)$

$v_{2}^{2}=2 W_{\mathrm{B}} y /\left(m_{\mathrm{B}}+I_{\mathrm{G}} / \mathrm{r}^{2}\right)$

$v_{2}=\left(2 W_{\mathrm{B}} y /\left(m_{\mathrm{B}}+I_{\mathrm{G}} / \mathrm{r}^{2}\right)\right)^{1 / 2}$

Kinematics With Constant Acceleration

$$
\begin{aligned}
& v_{2}^{2}=v_{1}^{2}+2 a\left(y_{2}-y_{1}\right) \\
& v_{2}^{2}=0+2 a y \\
& a=v_{2}^{2} / 2 y \\
& a=\left(2 W_{\mathrm{B}} y /\left(m_{\mathrm{B}}+I_{\mathrm{G}} / \mathrm{r}^{2}\right)\right) / 2 y \\
& a=\frac{W_{\mathrm{B}}}{\left(m_{\mathrm{B}}+\frac{I_{\mathrm{G}}}{r^{2}}\right)}
\end{aligned}
$$

$$
\begin{aligned}
& v_{2}=v_{1}+a t \\
& \left(2 W_{\mathrm{B}} y /\left(m_{\mathrm{B}}+I_{\mathrm{G}} / \mathrm{r}^{2}\right)\right)^{1 / 2}=0+W_{\mathrm{B}} /\left(m_{\mathrm{B}}+I_{\mathrm{G}} / \mathrm{r}^{2}\right) t \\
& t=\left[\left(2 W_{\mathrm{B}} y /\left(m_{\mathrm{B}}+I_{\mathrm{G}} / \mathrm{r}^{2}\right)\right)^{1 / 2}\right] /\left[W_{\mathrm{B}} /\left(m_{\mathrm{B}}+\right.\right. \\
& \left.\left.I_{\mathrm{G}} / \mathrm{r}^{2}\right)\right] \\
& t=\left[\frac{2 y\left(m_{\mathrm{B}}+\frac{I_{\mathrm{G}}}{r^{2}}\right)}{W_{\mathrm{B}}}\right]^{\frac{1}{2}} \leftarrow
\end{aligned}
$$


$\therefore$ Time is a function of wheel's inertia!

b)

$\underline{\text { FBD }}$

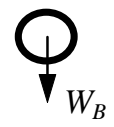

$\underline{\text { Kinematic Diagram }}$

○ $V_{2}$

$T_{1}+\Sigma U_{1-2}=T_{2}$

$1 / 2 m_{\mathrm{B}} v_{1}^{2}+U_{\mathrm{WB}}=1 / 2 m_{\mathrm{B}} v_{2}^{2} \quad \leftarrow U_{\mathrm{WB}}=W_{\mathrm{B}} y$

$0+W_{\mathrm{B}} y=1 / 2 m_{\mathrm{B}} v_{2}^{2}$

$2 W_{\mathrm{B}} y=m_{\mathrm{B}} v_{2}^{2}$

$v_{2}^{2}=2 W_{\mathrm{B}} y / m_{\mathrm{B}}$

$v_{2}=\left(2 W_{\mathrm{B}} y / m_{\mathrm{B}}\right)^{1 / 2}$

$\underline{\text { Kinematics With Constant Acceleration }}$

$$
\begin{aligned}
& v_{2}{ }^{2}=v_{1}{ }^{2}+2 a\left(y_{2}-y_{1}\right) \\
& v_{2}{ }^{2}=0+2 a y \\
& a=v_{2}^{2} / 2 y \\
& a=\left(2 W_{\mathrm{B}} y / m_{\mathrm{B}}\right) / 2 y \\
& a=\frac{W_{\mathrm{B}}}{m_{\mathrm{B}}}
\end{aligned}
$$

$$
\begin{aligned}
& v_{2}=v_{1}+a t \\
& \left(2 W_{\mathrm{B}} y / m_{\mathrm{B}}\right)^{1 / 2}=0+\left(W_{\mathrm{B}} / m_{\mathrm{B}}\right) t \\
& t=\left[\left(2 W_{\mathrm{B}} y / m_{\mathrm{B}}\right)^{1 / 2}\right] /\left[W_{\mathrm{B}} / m_{\mathrm{B}}\right] \\
& t=\left(2 y m_{\mathrm{B}} / W_{\mathrm{B}}\right)^{1 / 2} \\
& t=\left[\frac{2 y m_{\mathrm{B}}}{W_{\mathrm{B}}}\right]^{\frac{1}{2}} \leftarrow
\end{aligned}
$$

$\therefore$ Time for free fall will always be less than if ball is attached to wheel! Inertia of wheel decreases the ball's acceleration. 


\section{RIGID BODY: CONSERVATION OF MOMENTUM - ROTATING BICYCLE WHEEL}

Supplies: Bicycle wheel, four fishing weights, and string. The weights slide on the spokes and are connected to string that is threaded down through the center of the axle. The weights are moved towards the hub by pulling on the string.

Given: A bicycle wheel has weights attached from the spokes. The weights initially are located against the rim. The wheel is spinning with its axle in the vertical direction. The weights are then pulled towards the hub.

$I_{\mathrm{B}}=$ bicycle moment of inertia about axle

$m=$ mass of one weight

$r_{1}=$ radius to weights at rim

$r_{2}=$ radius to weights at hub

$\omega_{1}=$ angular velocity with weight at $r_{1}$

$\omega_{2}=$ angular velocity with weight at $r_{2}$

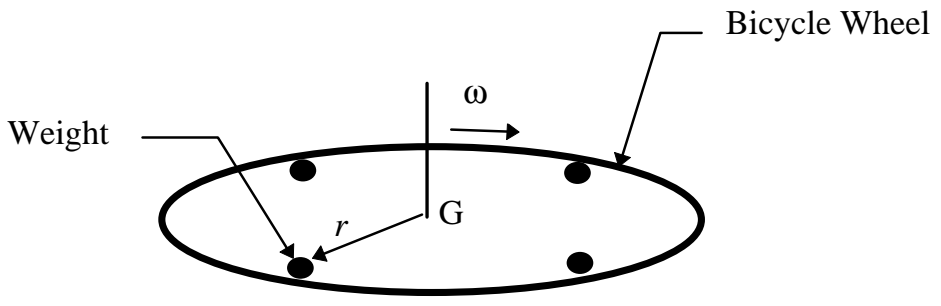

Find: What will happen to the wheel when the weights are pulled towards the hub?

\section{Solution:}

Momentum 1(top view)

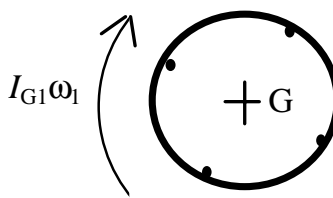

$\sum\left(H_{\mathrm{Z}}\right)_{1}+\sum \int M \mathrm{~d} t=\sum\left(H_{\mathrm{Z}}\right)_{2}$

$\left(I_{\mathrm{G}} \omega\right)_{1}+\sum \int M \mathrm{~d} t=\left(I_{\mathrm{G}} \omega\right)_{2}$

$I_{\mathrm{G} 1} \omega_{1}+0=I_{\mathrm{G} 2} \omega_{2}$

$\omega_{2}=\frac{\left(I_{\mathrm{B}}+4 m r_{1}^{2}\right)}{\left(I_{\mathrm{B}}+4 m r_{2}^{2}\right)} \omega_{1}$
$\left(I_{\mathrm{B}}+4 m r_{1}^{2}\right) \omega_{1}=\left(I_{\mathrm{B}}+4 m r_{2}^{2}\right) \omega_{2}$

$\underline{\text { Impulse(top view) }}$

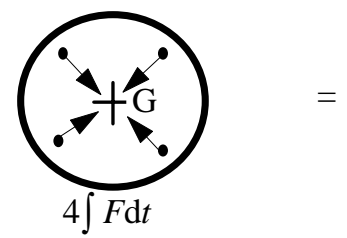

$\leftarrow \sum \int M \mathrm{~d} t=0$, the angular impulse is zero.

$\leftarrow I_{\mathrm{G}}=\left(I_{\mathrm{B}}+4 m r^{2}\right)$

$\therefore$ The angular velocity will increase because $r_{1}$ is greater than $r_{2} !$ ! The total moment of inertia is greater when the weights are at the rim.
Momentum 2(top view)

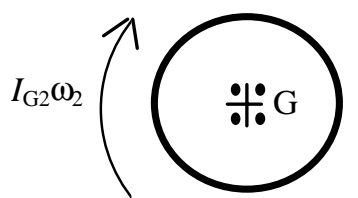

